\title{
Genome-wide sexually antagonistic variants reveal longstanding constraints on sexual dimorphism in the fruitfly
}

5

Filip Ruzicka ${ }^{1 \#}$, Mark S. Hill11,2\#, Tanya M. Pennell ${ }^{3}$, llona Flis ${ }^{4}$, Fiona C. Ingleby ${ }^{5}$, Kevin Fowler ${ }^{1}$, Edward H. Morrow ${ }^{5 \$^{*},}$, Max Reuter ${ }^{1 \$,{ }^{*}}$

$\#, \S$ Equal contributions

${ }^{*}$ Corresponding authors

151 Research Department of Genetics, Evolution and Environment, University College London, UK

${ }^{2}$ Department of Ecology and Evolutionary Biology, The University of Michigan, Ann Arbor, USA

20

${ }^{3}$ Daphne du Maurier Building, University of Exeter, Penryn, UK

${ }^{4}$ The Pirbright Institute, Pirbright, Surrey, UK

${ }^{5}$ School of Life Sciences, University of Sussex, Brighton, UK 
The evolution of sexual dimorphism is constrained by a shared genome, leading to 'sexual antagonism' where different alleles at given loci are favoured by selection in males and females. Despite its wide taxonomic incidence, we know little about the identity, genomic location and evolutionary dynamics of antagonistic genetic variants. To address these deficits, we use sex-specific fitness data from 202 fully sequenced hemiclonal $D$. melanogaster fly lines to perform a genome-wide association study of sexual antagonism. We identify $\sim 230$ chromosomal clusters of candidate antagonistic SNPs. In contradiction to classic theory, we find no clear evidence that the $X$ chromosome is a hotspot for sexually antagonistic variation. Characterising antagonistic SNPs functionally, we find a large excess of missense variants but little enrichment in terms of gene function. We also assess the evolutionary persistence of antagonistic variants by examining extant polymorphism in wild D. melanogaster populations. Remarkably, antagonistic variants are associated with multiple signatures of balancing selection across the $D$. melanogaster distribution range, indicating widespread and evolutionarily persistent (>10,000 years) genomic constraints. Based on our results, we propose that antagonistic variation accumulates due to constraints on the resolution of sexual conflict over protein coding sequences, thus contributing to the long-term maintenance of heritable fitness variation. 
The divergent reproductive roles of males and females favour different phenotypes $^{1,2}$. However, responses to these selective pressures are constrained by a shared genome, leading to 'sexual antagonism' where different alleles at given loci are favoured in the two sexes ${ }^{1,3-5}$. A wealth of quantitative genetic studies has established sexual antagonism as near ubiquitous across a wide range of taxa, including mammals ${ }^{6}$ (and humans ${ }^{7}$ ), birds ${ }^{8}$, reptiles ${ }^{9}$, insects ${ }^{10,11}$, fish ${ }^{12,13}$ and plants $^{14}$. Accordingly, sexual antagonism can be considered a major constraint on adaption and an important mechanism for the maintenance of fitness variation within populations ${ }^{15}$.

However, despite its evolutionary importance, we have little understanding of the biological mechanisms underlying this conflict and virtually no empirical data on the identity and evolutionary dynamics of antagonistic alleles ${ }^{13}$. While a small number of individual antagonistic loci have been identified ${ }^{12,13}$, these are of limited use for elucidating general properties of loci experiencing sexual antagonism. On a genomewide scale, previous transcriptomic work in D. melanogaster has associated antagonistic fitness effects with patterns of gene expression ${ }^{16}$. But despite potentially revealing some of the molecular correlates of fitness variation, this approach cannot distinguish between causal antagonistic loci and their downstream regulatory targets. In humans, genome-wide frequency differences between males and females have been used to infer sexually antagonistic selection on viability ${ }^{17}$, but this approach neglects important reproductive components of fitness. It is essential that we characterise causal antagonistic loci underlying lifetime reproductive success in order to understand the adaptive limits to sexual dimorphism and mechanisms of conflict resolution.

To address this shortcoming, we identified sexually antagonistic loci across the $D$. melanogaster genome and characterised their functional and evolutionary properties. Specifically, we obtained male and female fitness data for over 200 hemiclonal lines that had been extracted from $\mathrm{LH}_{\mathrm{M}}$, the outbred, laboratory-adapted population in which sexually antagonistic fitness effects were first characterised ${ }^{10,18}$. Our fitness measurements estimate lifetime reproductive success in both sexes by replicating the regime under which $\mathrm{LH}_{\mathrm{M}}$ has been maintained for over twenty years ${ }^{19}$. We combined these fitness data with high-coverage genome sequences ${ }^{20}$ and 
performed a genome-wide-association-study (GWAS) to map the genetic basis of sexual antagonism. We then examined the properties of candidate antagonistic polymorphisms, including their genomic distribution across the $\mathrm{X}$ chromosome and autosomes, the functional characteristics of candidate polymorphisms and the genes in which they occur, and their population genomic dynamics across a number of wild D. melanogaster populations.

\section{Results}

Quantitative genetic analyses confirmed the presence of significant amounts of genetic variation for male and female fitness among the lines assayed $(\mathrm{N}=223)$. Estimating the genetic variances and covariances between the lines, we found appreciable heritabilities for fitness in both sexes (female $h^{2}=0.42,95 \% \mathrm{Cl} 0.30$ $0.54 ;$ male $\left.h^{2}=0.16,95 \% \mathrm{Cl} 0.04-0.27\right)$. Comparable estimates were also obtained by treating single nucleotide polymorphisms (SNPs) as random effects in a linear

100 mixed model and calculating SNP heritability $\left(h_{S N P}^{2}\right)$ using restricted maximum likelihood (female $h_{S N P}^{2}=0.59, \mathrm{SD} 0.13, \mathrm{P}<0.001$; male $h_{S N P}^{2}=0.29$, SD 0.16, $\mathrm{P}=0.007)$. Overall, the intersexual genetic correlation for fitness did not differ significantly from zero in this sample of genotypes $\left(r_{M F}=0.15,95 \% \mathrm{Cl}-0.21-0.46\right)$. The presence of ample heritable fitness variation, combined with the lack of a strong 105 positive intersexual genetic correlation for fitness, suggests the presence of sexually antagonistic fitness variants.

We quantified antagonistic fitness variation by calculating an 'antagonism index'. Specifically, we rotated the coordinate system of the male and female fitness plane by 45 degrees and extracted the position of individual fly lines on the axis ranging from extremely male-beneficial, female-detrimental (MB) fitness effects to extremely female-beneficial, male-detrimental (FB) fitness effects (Fig. 1A). This approach for defining an antagonism index is analogous to other linear transformations, such as the widely applied transformation of human height and weight into a Body Mass Index ${ }^{21}$. The antagonism index itself had high SNP heritability $\left(h_{S N P}^{2}=0.51\right.$, SD 0.15 , $P=0.001$ ), as expected from the heritability of its sex-specific fitness components. 
To identify putative antagonistic SNPs, we performed a genome-wide association study (GWAS) based on the antagonism index and sequence polymorphism data for 765,654 common (MAF>0.05) and stringently quality-filtered SNPs across 202 of the 223 lines (see Methods; Sup. Fig. 1). We employed a linear mixed model that corrects for between-line relatedness and population structure by incorporating a genetic similarity matrix as a random effect ${ }^{22}$ (Sup. Fig. 2). Figure 1B presents a Manhattan plot of raw P-values from SNP-wise association tests along the $D$. melanogaster genome.

Although no individual SNP reached genome-wide significance based on stringent Bonferroni correction $\left(\mathrm{P}<6.53 \times 10^{-8}\right)$, our focus was to characterise broad patterns associated with genome-wide antagonistic variation rather than identifying individual

130 antagonistic sites with high confidence. Accordingly, we applied three main approaches to investigate the general properties of antagonistic SNPs and regions. First, we defined 2,372 candidate antagonistic SNPs (henceforth 'antagonistic SNPs') as SNP positions with false discovery rate (FDR) Q-values $<0.3$ (but note that the $Q$-values we estimated are likely to be conservative, see Sup. Fig. 2). This threshold achieves a balance between false positives and false negatives that is suitable for genome-wide analyse ${ }^{23}$ and allowed us to contrast the properties of antagonistic and non-antagonistic (Q-value $\geq 0.3$ ) SNPs. Second, we quantified the importance of different classes of SNPs (defined by chromosomal location or function) by partitioning total SNP heritability of the antagonism index ('antagonistic $h_{S N P}^{2}$ ') into the contribution of each class ${ }^{24,25}$. These contributions can then be tested for deviations from random expectations and interpreted without need for defining significance cut-offs for individual SNPs. Finally, we employed set-based association testing where the joint effect of a set of SNPs (such as those in a chromosomal window) on the phenotype is assessed. This joint analysis alleviates the multiple testing burden and can be used to define antagonistic windows with more stringent support $(Q$-value<0.1). Together these approaches allowed us to characterise the functional properties and evolutionary dynamics of antagonistic genetic variation.

We first examined the genomic distribution of antagonistic variants. The 2,372 antagonistic SNPs were significantly clustered along chromosome arms (median 
distance: $147 \mathrm{bp}$ on autosomes, $298 \mathrm{bp}$ on the $\mathrm{X}$ chromosome, permutation test: $P<0.001$ for autosomes and $X$, Sup. Fig. 3). Using LD clumping ${ }^{26}$, we estimated that the antagonistic SNPs form approximately 226 independent clusters. Some previous theory ${ }^{3}$ and empirical quantitative genetic results ${ }^{17}$ suggest that the $X$ chromosome should harbour a disproportionate amount of antagonistic genetic variation. This was not borne out by our data. We found that, relative to autosomes, the $\mathrm{X}$ chromosome neither contained a disproportionate number of antagonistic SNPs (Z-test, P>0.05, Sup. Fig. 4), nor contributed more antagonistic $h_{S N P}^{2}$ than expected (Fig. 2A).

160 Our data also allowed us to provide some of the first insights into the biological functions that underlie sexual antagonism. At the most basic level, our results suggest that antagonism arises primarily due to adaptive conflict over coding sequences. Thus, genomic partitioning revealed that variants which result in missense changes were significantly over-represented among antagonistic SNPs

165 (Sup. Fig. 4) and contributed significantly more antagonistic $h_{S N P}^{2}$ than expected from their proportional genomic representation (Fig. 2A; Sup. Tab. 1). As expected, intergenic regions were under-represented among antagonistic SNPs and contributed qualitatively less antagonistic $h_{S N P}^{2}$ than expected (Fig. 2A; Sup. Fig. 4). However, we found no evidence that SNP functions involved in expression regulation, such as 3'UTR, intronic, upstream or splice region variants, were overrepresented among antagonistic SNPs or $h_{S N P}^{2}$ (Fig. 2A; Sup. Fig. 4).

We next performed a series of analyses to characterise the properties of genes harbouring antagonistic SNPs (one or more antagonistic SNPs within $\pm 5 \mathrm{~Kb}$ of the gene coordinates). The list of antagonistic genes included some genes known to be involved in sexual differentiation, including male-specific-lethal-1, traffic jam, and roundabout 2, the circadian clock gene period, and the Golgi-associated transport protein gene Tango6 that has been previously found to harbour coding sequence polymorphism shared between $D$. melanogaster and D. simulans ${ }^{27}$ (see Sup. Tab. 2 180 for a complete list of antagonistic genes). Reflecting the heterogeneous list of genes, Gene Ontology (GO) analysis revealed little evidence for preferential association of antagonistic variation with specific biological processes. Only one term, 'sodiumchannel-regulator-activity', was significant after correction for multiple-testing (Q- 
value $=0.013)$. However, this annotation is shared by only a few genes $(\mathrm{N}=5)$, a cluster of four of which carry antagonistic SNPs. It thus appears that antagonism is not enriched in genes involved in specific functions.

While antagonistic genes were not enriched in specific functions, they did show lower than average sex-bias in gene expression. This pattern is expected because

190 unbiased genes should be most prone to experiencing balanced, opposing selection pressures in the two sexes that would stabilise antagonistic variation. We found evidence for this enrichment both in qualitative terms, where fewer antagonistic genes than expected by chance showed significant sex-biased gene expression (observed $=188$, expected $=212,11.3 \%$ deficit, $\chi_{1}^{2}=7.78, P=0.005$ ), and in quantitative

195 terms, where antagonistic genes had a lower degree of sex-bias than did nonantagonistic genes $(\mathrm{W}=1309700, \mathrm{P}<0.001$, Fig. 2B). We did not, however, detect significant overlap between the antagonistic genes identified here and genes that had previously been shown to have sexually antagonistic expression patterns (opposing relationships between expression level and fitness in males and

200 females $^{16}$, observed number of overlapping genes $=41$, expected number $=36$, $\left.\chi_{1}^{2}=0.59, \mathrm{P}=0.44\right)$. This discrepancy is not unexpected, as the causal genetic changes underlying antagonism need not primarily be associated with expression differences (as suggested by enrichment among missense variants), nor need genes showing expression divergence between the phenotypic fitness extremes

205 necessarily carry causal genetic variants themselves. Finally, we tested whether antagonistic variation is enriched in genes that are likely to be subject to pleiotropic constraints, as this has been proposed to make sexual antagonism harder to resolve $^{28}$. We did not find an association between antagonism and higher levels of pleiotropy, measured either as tissue-specificity ${ }^{29}(\tau: W=2319200, P=0.80)$ or as the

210 number of protein-protein interactions ${ }^{30}$ (PPIs: $F_{1,5276}=2.43, P=0.12$ ). This implies that pleiotropy-at least as captured by $\tau$ and PPIs-does not contribute significantly to maintaining sexually antagonistic genetic variation.

In addition to assessing the functional properties of antagonistic loci, we also investigated the population genetic effects of sexual antagonism. Models predict that the opposing sex-specific fitness effects of antagonistic alleles generate balancing 
selection, resulting in elevated levels of genetic polymorphism at antagonistic loci ${ }^{31-}$ 33. Having identified putatively antagonistic variants, we can test this prediction by comparing levels of polymorphism at antagonistic and non-antagonistic loci. Doing so directly within the $\mathrm{LH}_{\mathrm{M}}$ population is problematic, because the power to detect antagonistic effects is higher at more polymorphic sites, and candidates therefore tend to show above-average polymorphism. However, we can use data from independent populations and ask whether, for a given level of polymorphism in $\mathrm{LH}_{\mathrm{M}}$, polymorphism there is greater at antagonistic than at non-antagonistic sites. We performed this type of analysis (see Methods for details) using publicly available polymorphism data from the Drosophila Genetics Reference Panel ${ }^{34,35}$ (DGRP), a collection of 205 wild-derived inbred lines. Like LH $_{M}$, the DGRP was established from a North American $D$. melanogaster population. Given the relatively recent colonisation of the continent by $D$. melanogaster ( $\sim 150$ years $\left.^{36}\right)$, the two populations

230 are closely related. We found that antagonistic SNPs had elevated minor allele frequencies (MAFs) in the DGRP, although owing to the close relationship between $\mathrm{LH}_{\mathrm{M}}$ and the DGRP (and the resulting similarity in allele frequencies) this difference was not statistically significant $(P=0.322$, Fig. 3A,B). However, when using the $P$ value for the antagonistic effects of individual SNPs rather than a binary antagonistic/non-antagonistic categorisation of sites, we found a significant negative correlation between P-value and MAF in the DGRP, consistent with elevated polymorphism in the DGRP at SNPs that are more closely associated with antagonism in $\mathrm{LH}_{\mathrm{M}}(\rho=-0.055, \mathrm{P}=0.044$, Fig. $3 \mathrm{C})$. This evidence for antagonismdriven balancing selection at individual sites was corroborated by patterns of regional 240 polymorphism-measured as Tajima's D within 1000bp windows along the chromosome arms. Tajima's D was significantly higher in antagonistic windows (those with Q-value<0.1 in a window-based GWAS) than in non-antagonistic windows (Q-value $\geq 0.1 ; F_{1,115477}=224.6, P<0.001$, Fig. 3G). Overall, these analyses show that the heritable phenotypic variation in sex-specific fitness that can be generated and maintained by sexual antagonism is mirrored by a signal of increased polymorphism at the underlying genetic loci.

A key, yet so far unresolved question is whether antagonistic polymorphisms are mainly short-lived and population-specific or persist over prolonged periods of time. 
The analyses of polymorphism in the DGRP shed some light on this question, demonstrating that antagonistic polymorphisms are maintained at least over periods of tens to hundreds of years, or hundreds to a few thousand generations. In order to assess signals of balancing selection over longer time spans, we repeated these analyses with data from a population in $D$. melanogaster's ancestral Sub-Saharan distribution range, in Zambia (ZI, 197 genomes from phase 3 of the Drosophila Population Genomics Project ${ }^{37}$; see also Sup. Fig. 5, which repeats the $\mathrm{ZI}$ analyses with identical results using 118 genomes from South Africa ${ }^{38}$ ). Just as in the DGRP, we found that antagonism generated a clear signature of balancing selection in this ancestral population sample. Analyses based on binary categories showed that antagonistic SNPs had significantly higher MAFs in ZI compared to non-antagonistic SNPs ( $P=0.024$, Fig. 3D,E), while analyses based on $P$-values showed again that sites with stronger evidence for antagonistic effects had more elevated MAFs $(\rho=-0.070, \mathrm{P}=0.002$, Fig. 3F). At a larger chromosomal scale, antagonistic windows had significantly higher polymorphism (Tajima's D) than non-antagonistic windows

$265\left(F_{1,116099}=60.63, P<0.001\right.$, Fig. 3G). Furthermore, they also exhibited lower population differentiation between DGRP and ZI (measured as Fst; Wilcoxon RankSum test, $W=63416000, P=0.012$, Fig. 3H; Sup. Fig. 5), in line with balancing selection maintaining similar frequencies across distant populations.

270 In addition to elevated polymorphism in antagonistic regions of the genome, we also found evidence for increased linkage disequilibrium (LD) - another hallmark of balancing selection ${ }^{39}$. We compared local LD $\left(<1,000 \mathrm{bp}\right.$, measured as $\left.\mathrm{r}^{2}\right)$ between pairs of antagonistic sites, pairs of non-antagonistic sites, and 'mixed' site pairs (consisting of an antagonistic and a non-antagonistic SNP) in the ZI population,

275 which is most phylogenetically distant from $\mathrm{LH}_{\mathrm{M}}$ and where a signal of LD should be weakest in the absence of long-term balancing selection. Consistent with selection, we found that pairs of antagonistic sites had higher LD in this population than pairs of non-antagonistic sites (Wilcoxon Rank-Sum tests, W=8346500000, $P<0.001$, Fig. 3I). They also had higher LD relative to mixed pairs (Wilcoxon Rank-Sum test, $W=33823000, P<0.001$, Fig. 3I). Thus, high LD between antagonistic sites is not an artefact of unusually low levels of recombination near antagonistic regions, but instead reflects the action of long-term balancing selection. 
Taken together, these comparative population genomic analyses demonstrate that the antagonistic allelic variation identified in $\mathrm{LH}_{\mathrm{M}}$ is neither recent nor populationspecific. To a significant degree, balancing selection maintains antagonistic variation over timescales that extend beyond the extension of the species range out of Africa, more than 10,000 years ago ${ }^{36}$.

\section{Discussion}

Our study provides the first genome-wide analysis of the identity, function and evolution of sexually antagonistic sequence polymorphisms in fruitflies. Remarkably, we find that genetic variation at antagonistic loci is stably maintained across $D$. melanogaster populations throughout the species' distribution range, indicating that the targets of antagonistic selection have been largely conserved for many millennia $36,40-42$ - and several tens of thousands of generations. The geographical stability and low turnover in antagonistic sequence variation implies that adaptive conflict between males and females is rooted in a fundamental aspect of the biology of the sexes and persists even in the face of environmental variation ${ }^{43}$. It is therefore unaffected by the adaptation of populations to the environmental conditions that they encountered during their colonisation of the globe ${ }^{36,41,44}$ or the continuous adaptive evolution that occurs within temperate populations over the course of the seasons ${ }^{23}$. More generally, our results supplement a growing body of evidence ${ }^{23}$ suggesting that balancing selection can influence patterns of genetic variation on a genome-wide scale, rather than simply a small number of isolated $l o \mathrm{l}^{45}$, as is often assumed ${ }^{46}$. Sexually antagonistic selection should contribute particularly strongly to the build-up of balanced polymorphisms, given that there is abundant evidence for sex-specific selection in nature ${ }^{4,47}$ and that sex-specific selection can generate permissive conditions for the evolution of such polymorphisms relative to alternative modes of balancing selection ${ }^{33,48}$.

The long-term stability of sexually antagonistic polymorphisms further suggests that the evolutionary constraints on sexual dimorphism inherent in antagonism are difficult to resolve. While we do not find any evidence that there is elevated pleiotropy among genes experiencing ongoing conflict, the persistence of antagonism fits with our finding that antagonistic polymorphisms are highly enriched 
for missense variants. While antagonistic selection on expression levels can be accommodated by gradual evolution of sex-specific gene expression ${ }^{49}$, adaptive conflicts over coding sequences can only be resolved through a complex multi-step process $^{50}$ of gene duplication, sex-specific sub-functionalisation of coding sequences and the evolution of differential expression of the two paralogues. The requirement for gene duplication, in particular, would be expected to constitute a severely limiting barrier for this route towards resolution, as suitable mutation events will be exceedingly rare. This large barrier to resolution, and the resulting stochasticity in which antagonisms will undergo resolution, may help to explain the lack of GO enrichment observed among antagonistic genes.

We find no convincing evidence that the $\mathrm{X}$ chromosome is enriched for antagonistic variation, in contradiction to classic theory ${ }^{3}$. This discrepancy could be due to the 330 presence of dominance reversal, where the beneficial allele is dominant in each sex. Such sex specific dominance has recently been documented empirically ${ }^{13}$ and is predicted to shift enrichment of antagonism from the $X$ to the autosomes ${ }^{51}$ _ particularly so if antagonistic loci interact epistatically ${ }^{52}$. Furthermore, the hemiclonal approach might miss low-frequency X-linked antagonistic polymorphisms with recessive fitness effects, as these will rarely be expressed in phenotypic assays. However, while these general effects might explain the lack of $\mathrm{X}$-enrichment, our result also contradicts previous empirical findings obtained in the $\mathrm{LH}_{\mathrm{M}}$ population ${ }^{17}$ itself, which found that the $\mathrm{X}$ chromosome contributed disproportionally to antagonistic fitness variation. The previous study was based on a much smaller sample of genomes, with large uncertainties about the estimated chromosomal contributions. It was also performed more than ten years ago and much closer to the establishment of $\mathrm{LH}_{\mathrm{M}}$ as a laboratory population. Accordingly, the discrepancy to our results might in part be explained by stronger genetic drift on the $\mathrm{X}$ chromosome relative to autosomes, which could in turn lead to a disproportionate loss of X-linked antagonistic polymorphisms ${ }^{32}$.

Taken together, this study addresses a longstanding gap in our understanding of sexual antagonism, and provides a valuable resource from which to further elucidate the origin and resolution of this fundamental evolutionary phenomenon. 


\section{Methods}

\section{$\mathrm{LH}_{\mathrm{M}}$ hemiclones}

$\mathrm{LH}_{\mathrm{M}}$ is a laboratory-adapted population of Drosophila melanogaster that has been maintained under a highly controlled rearing regime since $1996^{53}$. A random sample of 223 genetic lines was created from the population ${ }^{20}$ using a hemiclonal approach $^{54}$. Individuals of each line carry an identical haploid genome comprising the major chromosomes X, 2 and 3. Crosses with flies from custom stocks allows the generation of many replicate individuals - males and females - that carry a line's X2-3 haplotype alongside a random chromosomal complement from the $\mathrm{LH}_{M}$ population that can be assayed for fitness.

\section{Fitness measurements}

Lifetime adult reproductive fitness of males and females of each line was measured using assays designed to mimic the $\mathrm{LH}_{\mathrm{M}}$ rearing regime. For male fitness, we measured competitive fertilisation success by setting up competition vials containing

3655 hemiclonal males from a given line, 10 competitor bw males and 15 virgin bw females. After two days, bw females were isolated into individual vials containing no additional yeast and left to oviposit for 18 hours. On day 12 post egg-laying, progeny were scored for eye colour. Male fitness was calculated as the proportion of offspring sired by the 5 hemiclonal males (those with wildtype eye-colour), combining progeny data from the 15 oviposition vials. This assay was repeated 5 times in a blocked design; estimates for each line were therefore based on fitness measurements from 25 hemiclonal males.

Female fitness was measured as competitive fecundity. Competition vials containing 5 virgin hemiclonal females from a given line, 10 competitor bw females and $15 \mathrm{bw}$ males were set up. Two days later, the 5 hemiclonal females were isolated into individual vials and left to oviposit for 18 hours. These vials were immediately chilled at $4^{\circ} \mathrm{C}$ and fecundity was measured by counting the number of eggs laid per female. This assay was replicated 5 times in a blocked design; each line estimate therefore measured the fitness of 25 hemiclonal females. preparation for quantitative genetic and association analysis. Male fitness data from competition vials where not all 5 focal males were present at the end of the assay 
were removed from further analysis. Similarly, we omitted female oviposition vials where fewer than 2 eggs were present (indicating partial sterility or failure to mate) or measurements were then first box-cox transformed to be normally distributed within each block, then scaled and centred. To calculate SNP heritabilities and for association analysis, data from each block were averaged to obtain one fitness estimate for each line and sex.

390

\section{Quality control of whole-genome sequences}

We used previously published whole genome sequences generated from the hemiclonal lines analysed here ${ }^{20}$. Details about DNA extraction, library preparation, sequencing, read processing and SNP calling are provided in the original publication.

395 Prior to the association analysis performed here, further site-level quality filtering steps were performed in vcftools ${ }^{55}$ and $P L I N K^{26}$. First, individual variant calls based on depth $<10$ and genotype quality $<30$ were removed. Second, individuals with $>15 \%$ missing positions were removed. Third, positions with poor genotype information ( $<95 \%$ call rate) across all retained individuals were discarded. Finally, given the relatively small sample size of the dataset as a whole and the low power of an association test for rare variants, we retained only common variants (MAF>0.05) for further analysis. From an initial dataset of 220 hemiclones containing 1,312,336 SNPs, this yielded a quality-filtered dataset of 765,980 SNPs from 203 hemiclones.

To detect outliers, we examined $\mathrm{LH}_{\mathrm{M}}$ 's population structure using principal components analysis (PCA). Overlapping SNP positions from the $203 \mathrm{LH}_{\mathrm{M}}$ genomes and from an outgroup population (Drosophila Genetic Reference Panel, or DGRP ${ }^{34}$ ) consisting of 205 whole-genome sequenced individuals were used as input to construct a genetic similarity matrix. This set of SNPs was pruned for linkage disequilibrium (LD) such that no two SNPs with $r^{2}>0.2$ within $10 \mathrm{~Kb}$ remained. The

410 leading PC axes were extracted in LDAK ("Linkage-Disequilibrium Adjusted Kinships"56). After removal of one outlier (see Sup. Fig. 1A), the final dataset used for association analysis contained 202 individuals and 765,764 SNPs.

\section{Heritability analyses}

415 We estimated the variance-covariance matrix for fitness and sex-specific residual variances by fitting a model using $\mathrm{MCMCglmm}^{57}$ implemented in R. Specifically, we 
fitted the model $Y_{i j k}=X_{i j}+\varepsilon_{i j k}$, where $Y_{i j k}$ is the scaled and centred fitness of individual $\mathrm{k}$ of genotype $\mathrm{j}$ and sex $\mathrm{i}, X_{i j}$ is the sex-specific random effect of genotype $\mathrm{j}$ in sex $\mathrm{i}$, and $\varepsilon_{i j k}$ describes the sex-, genotype- and individual-specific residual. The genotypic fitness effects in males and females follow a bivariate normal distribution $X_{i j} \sim N(0, \mathrm{G})$, where

$$
G=\left(\begin{array}{cc}
\sigma_{G, m}^{2} & \operatorname{Cov}_{G, m f} \\
\operatorname{Cov}_{G, m f} & \sigma_{G, f}^{2}
\end{array}\right)
$$

is the genetic variance-covariance matrix across sexes (composed of male and female additive genetic variances $\sigma_{G, m}^{2}$ and $\sigma_{G, f}^{2}$ and the intersexual genetic

425 covariance $\left.\operatorname{Cov}_{G, m f}\right)$. Residuals follow a normal distribution $\varepsilon_{i j k} \sim N\left(0, \sigma_{R, i}^{2}\right)$, where $\sigma_{R, i}^{2}$ is the sex-specific residual variance, and are assumed to be uncorrelated across sexes.

From these variance estimates, we calculated male and female heritabilities of fitness as $h_{i}^{2}=2 \sigma_{G, i}^{2} /\left(\sigma_{G, i}^{2}+\sigma_{R, i}^{2}\right)$, where the subscript $\mathrm{i}$ indicates either male or female. The factor 2 in the heritability calculation reflects the fact that with the hemiclonal approach, individuals assayed share half their genetic material (the hemizygous hemiclonal genome). The intersexual genetic correlation was calculated as $r_{m f}=\operatorname{Cov}_{G, m f} /\left(\sqrt{\sigma_{G, m}^{2}} \sqrt{\sigma_{G, f}^{2}}\right)$. The quantitative genetic parameters $h_{m}^{2}, h_{f}^{2}$ and $r_{m f}$ were calculated for each sample from the Monte Carlo Markov chain. From

435 these series of values we obtained point estimates (averages) and 95\% credible interval (using the function HPDintervals).

As a complementary approach, we estimated the SNP heritability $\left(h_{S N P}^{2}\right)$ of male and female fitness in $\operatorname{LDAK}^{56}$. This approach uses Restricted Maximum Likelihood (REML) ${ }^{58}$ to fit a linear mixed model that expresses the vector of 440 phenotypes $Y$ as a function genome-wide SNP genotypes, treated as random effects:

$$
Y \sim N\left(0, \sigma_{S N P}^{2} K+\sigma_{e}^{2} I\right)
$$

where $\mathrm{K}$ the kinship matrix, $\sigma_{S N P}^{2}$ a vector of additive genetic variances for each SNP, $\sigma_{e}^{2}$ the vector of residual variances and I an individual identity matrix. SNP heritability 445 is then estimated as $h_{S N P}^{2}=\sigma_{S N P}^{2} /\left(\sigma_{S N P}^{2}+\sigma_{e}^{2}\right)$.

LDAK corrects for local linkage when calculating SNP heritabilities to avoid inflation of $h_{S N P}^{2}$ in clusters of linked sites that otherwise arises because several 
SNPs tag the same causal polymorphism. SNPs are weighted inversely proportional to their local linkage, such that SNPs in high LD contribute less to $h_{S N P}^{2}$ than SNPs in low LD. This model has been shown to substantially improve heritability estimates across a wide range of traits ${ }^{24}$. LDAK also allows to set the parameter $\alpha$ that determines how SNPs are weighted by their MAF (as MAF $\left.{ }^{\alpha}\right)$ when calculating the kinship matrix K. We used the default of $\alpha=-0.25$ which provides a steeper relationship between MAF and $h_{S N P}^{2}$ than the value of -1 that is frequently used in

455 studies on humans. Significance of $h_{S N P}^{2}$ estimates was assessed by permuting phenotype labels 1,000 times, re-calculating $h_{S N P}^{2}$ on each permutation as above, and calculating the number of permuted estimates which exceeded the observed.

\section{Quantification and association analysis of sexual antagonism}

460 To identify loci underlying sexual antagonism, we defined an antagonism index (see main text, Fig. 1A). We calculated its SNP heritability ('antagonistic $h_{S N P}^{2}$ ') in LDAK, following the same procedure and settings as those for estimating sex-specific SNP heritabilities.

We performed a GWAS by applying a linear mixed model to test the effect of

465 allelic variants at each SNP on the antagonism index, while including the kinship matrix as a random effect to account for the heritable portion of genetic variation attributable to kinship between individuals. This approach has been shown to effectively control the false positive rate and increase power to detect true associations in samples with moderate degrees of population structure and close relatedness, such as $\mathrm{LH}_{\mathrm{M}}{ }^{22,59}$. The GWAS was implemented in LDAK (settings as above) and a Wald $\chi^{2}$ test was used to generate P-values for each position.

The genomic inflation factor ${ }^{60}$ of $\lambda_{\text {median }}=0.967$ (calculated as median $\chi^{2}$ obs $/$ median $\chi^{2} \exp$ in GenABEL ${ }^{61}$ in $\mathrm{R}$ ) suggests that genetic confounding has been well controlled in our GWAS. This is corroborated by the fact that the distribution of P-

475 values when permuting the phenotype labels (100 times and applying the same linear mixed model) was not enriched for low P-values (such a pattern would be expected if residual genetic confounding remained in our sample).

\section{Defining candidate antagonistic SNPs and regions}


480 We corrected for multiple testing using a False Discovery Rate (FDR) approach and converted P-values into Q-values. We defined antagonistic SNPs as sites with FDR Q-values $<0.3$ and non-antagonistic SNPs as sites with $Q$-value $\geq 0.3$.

For analyses which consider larger genomic regions (windows), we ran a setbased association test implemented in LDAK (options using '-calc-genes-reml',

485 'ignore-weights YES' and $\alpha=-0.25)$. The test calculates set-wide $h_{S N P}^{2}$ via REML, corrects for local relatedness using the predictors in each window, and computes a $P$-value using a likelihood ratio test (LRT). The sets we used were 1000bp windows (500bp step) defined according to Drosophila Reference 5 genome coordinates, and subsequently converted (using UCSC's liftOver tool ${ }^{62}$ ) to Release 6 coordinates.

490 This was a necessary step, as publicly available polymorphism data was mapped to Release 5 of the D. melanogaster genome, whereas the GWAS data was mapped to Release 6. We then calculated window-based Q-values from the LRT P-values and defined antagonistic windows as those with $Q$-value $<0.1$.

\section{Genomic distribution of antagonistic SNPs}

To estimate the number of independent antagonistic regions, we performed LDclumping in PLINK. We used a significance threshold of 0.00093 for the index SNP (the maximum, least significant, P-value across all antagonistic SNPs), and clustered ('clumped') neighbouring antagonistic SNPs by specifying an $r^{2}$ threshold of 0.4 and a distance threshold of $10 \mathrm{~Kb}$.

We also quantified the clustering by calculating the median distance between all pairs of adjacent antagonistic SNPs across chromosome arms. We did this separately for the autosomes and $\mathrm{X}$ chromosome, to accommodate for the lower SNP density on the $\mathrm{X}$ chromosome. We tested for significant clustering by using a

505 permutation test, where antagonistic/non-antagonistic labels was permuted among all SNPs, distances between adjacent SNPs labelled as 'antagonistic' after permutation were recalculated as before and the median distance recorded. This process was repeated 1,000 times in order to generate a null distribution of median distances. Significance of clustering among true antagonistic SNPs was calculated

510 as the proportion of median distances in the null distribution that were lower than or equal to the true median distance. 
To examine the proportional contribution of autosomal and X-linked antagonistic variants to total $h_{S N P}^{2}$, we used two complementary methods. First, we partitioned the genome into $X$ chromosome and autosome subsets, and calculated

$515 h_{S N P}^{2}$ via REML in LDAK each subset in turn (default parameters; $\alpha=-0.25$ ). The observed proportion of $h_{S N P}^{2}$ contributed by each compartment was then compared to the expected proportion (i.e., the fraction of LD-weighted predictors belonging to each compartment). We tested whether the two compartments contributed significantly more $h_{S N P}^{2}$ than expected using a two-sample Z-test. Second, we

520 compared the proportion of antagonistic SNPs $(Q-v a l u e<0.3)$ to the proportion of all SNPs mapping to each chromosomal compartment, using Z-tests. The under- or over-representation of antagonistic SNPs (deficit or excess of antagonistic compared to all SNPs) in each compartment is therefore unaffected by differences in SNP density between chromosome arms, such as the lower density on the $\mathrm{X}$ chromosome.

\section{Functional analyses of antagonistic loci}

We used the variant effect predictor (Ensembl VEP ${ }^{63}$ ) to map SNPs to functional categories. We partitioned total antagonistic $h_{S N P}^{2}$ into functional subsets, and estimated the observed proportion of $h_{S N P}^{2}$ contributed by each subset using REML in LDAK (settings as above). We then used a permutation test to compare observed and expected $h_{S N P}^{2}$ for each functional category, where we shifted genome-wide annotations to a random starting point along a 'circular genome'. This procedure breaks the relationship between each SNP and its annotation while preserving the order of annotations and their associated LD structure ${ }^{64} . h_{S N P}^{2}$ was re-calculated via REML for each of 1,000 permuted datasets and two-tailed P-values determined as the sum of permuted estimates with more extreme absolute values than the observed. As a complementary approach, we compared the proportion of antagonistic SNPs to the proportion of all SNPs mapping to each functional category. We then assessed enrichment for each functional category in turn using Z-tests.

We also used the VEP to map SNPs to genes. We included extended gene regions (+/- $5 \mathrm{~kb}$ of gene coordinates, VEP default) in our gene definition. To gain preliminary insights into the functions of antagonistic genes we used the Gorilla ${ }^{65}$ 
Gene Ontology tool, with FDR correction for multiple testing across GO terms. All genes covered in the final SNP dataset were used as the background set.

To examine the relationship between antagonistic genes and sex-biased gene expression we used the Sebida online database ${ }^{66}$ to annotate genes as having either sex-biased or unbiased expression profiles (meta-class identifier). We then used a $\chi^{2}$ test to compare the sex-biased expression status of antagonistic and nonantagonistic genes. We additionally examined the quantitative degree of sex-bias using this same dataset. We took the absolute value of the log2 transformed 'M_F' bias variable, such that large values indicate more extreme sex bias in expression irrespectively of whether this bias is towards males or females. We compared the distributions of this variable between antagonistic and non-antagonistic genes using

555 a Wilcoxon Rank-Sum test.

To assess the degree of overlap between antagonistic genes identified here and those associated with sexually antagonistic expression patterns in a previous study ${ }^{16}$, we included only genes covered in both datasets, and only those genes in both datasets that were adult-expressed. To determine whether genes were adult-

560 expressed we used the Drosophila gene expression atlas (FlyAtlas ${ }^{67}$ ).

Conservatively, we considered a gene 'adult-expressed' if its transcript was detected as present in at least one library of one adult-derived sample. We then used a $\chi^{2}$ test to assess the degree of overlap between the datasets.

We used the tissue-specificity index $(\tau)$ to compare pleiotropy between

565 antagonistic and non-antagonistic genes. We used gene expression data from FlyAtlas ${ }^{67}$ to get average expression values for each gene and in each tissue and then calculated $\tau$ as:

$\tau=\frac{\sum_{i=1}^{n}\left(1-\widehat{x_{l}}\right)}{n-1}$

where $\widehat{x_{l}=\frac{x_{i}}{\max \left(x_{i}\right)}}$ is the proportional expression level of the gene in tissue $i$ and $\bar{n}$

570 is the number of tissues. We compared values of $\tau$ for antagonistic and nonantagonist genes using a Wilcoxon rank-sum test.

As an additional proxy for pleiotropy we examined the number of proteinprotein interactions (PPIs) between antagonistic and non-antagonistic genes. We used the physical interactions table from FlyBase ${ }^{68}$ to summarise the total number of 575 PPIs for all genes and then compared candidate and non-candidate genes using a 
general linear model (GLM) with quasipoisson error structure to account for overdispersion.

\section{Comparative population genomic data}

580 To analyse SNP polymorphism outside the $\mathrm{LH}_{\mathrm{M}}$ population, we used publicly available population genomic data from three wild $D$. melanogaster populations. The first is an introduced population from North America (Drosophila Genetic Reference Panel ${ }^{34,35}$, denoted DGRP: 205 whole-genome sequences derived from inbred lines). The two others come from $D$. melanogaster's ancestral distribution range in subSaharan Africa (Zambia-ZI: 197 whole-genome sequences derived from haploid embryos; South African-SA: 118 whole-genome sequences derived from inbred lines, combines data from sub-populations 'SD' and 'SP', which have very low population differentiation $\left.{ }^{38}\right)$.

All genome sequences were downloaded as FASTA files from the Drosophila

590 Genome Nexus website (www.johnpool.net/genomes.html). These files had been generated following standardised alignment and quality filtering steps ${ }^{37}$ and were further quality-filtered for admixture and identity-by-descent using scripts provided on the Genome Nexus website. We used snp-sites ${ }^{69}$ to call SNPs and convert the multiple sequence alignments to vcf format. Allele frequencies in the three

595 populations were calculated using vcftools. We further excluded tri-allelic and poorly covered sites (call rate<20).

\section{SNP-based analyses of balancing selection}

To test whether antagonistic sites are associated with signatures of balancing selection, we closely followed the approach of Turchin et al. ${ }^{70}$ and looked for an increased minor allele frequency (MAF) at antagonistic relative to non-antagonistic sites (as identified in $\mathrm{LH}_{\mathrm{M}}$ ) in the three comparison populations (DGRP, ZI, SA). By focussing on the contrast between classes of SNPs, we ensured that demographic differences between populations did not confound our analyses.

We first LD-pruned the $\mathrm{LH}_{\mathrm{M}}$ dataset by clumping (in PLINK) to avoid pseudoreplication due to correlations between SNPs. For antagonistic sites, we used the 226 index SNPs identified in the previous clumping. For non-antagonistic sites, a non-antagonistic SNP was randomly chosen as an index SNP and clumped by clustering all SNPs within $10 \mathrm{~kb}$ with $\mathrm{r}^{2}>0.4$. Pruning in this manner reduced the 
original dataset of 765,764 SNPs to 36,319 "LD-independent" SNPs. For each of these SNPs, we then estimated MAFs in each comparison population. We assigned $M A F=0$ to sites which were monomorphic in a comparison population and those where a comparison population was polymorphic for variants other than those segregating at that site in the $\mathrm{LH}_{\mathrm{M}}$.

We then used this LD-independent dataset to compare MAF between antagonistic and non-antagonistic SNPs. We did this using a Monte Carlo approach where, 1,000 times, we paired the 226 antagonistic SNPs with 226 randomly drawn non-antagonistic "control" SNPs. The latter were carefully frequency-matched to the 226 antagonistic SNPs. The matching procedure first corrected $\mathrm{LH}_{\mathrm{M}} \mathrm{MAF}$ for 'linked selection'71 by taking the residuals of a linear regression of $\mathrm{LH}_{\mathrm{M}}$ MAF on estimates of linked selection. These estimates quantify local recombination rates and proximity to functional sequences in $D$. melanogaster. They thereby account for factors that affect polymorphism along the genome, such as background selection and selective sweeps. We then drew sets of 226 non-antagonistic SNPs to match the residual $\mathrm{LH}_{\mathrm{M}}$

625 MAF distribution of the 226 antagonistic SNPs and for each set calculated the mean MAF in the comparison population. The 1,000 sets generated in this way provided a null distribution of MAFs for non-antagonistic sites in each comparison population. Pvalues for deviations in polymorphism between antagonistic and non-antagonistic sites were then calculated by comparing, in each population, the mean MAF of the

630226 antagonistic SNPs to the null MAF distribution.

A second analysis used the same LD-independent dataset but considered the whole spectrum of P-values, rather than a binary split of SNPs into antagonistic/nonantagonistic categories. To this end, we performed binning in two dimensions, by residual $\mathrm{LH}_{\mathrm{M}}$ MAF (20 quantiles) and P-values (100 quantiles). We then drew one SNP from each of these MAF/P-value bins (2,000 SNPs in total), recorded the MAF for each in the comparison population of interest, and finally correlated these MAF values with P-values of the associated SNPs in the LHM using a Spearman's rank correlation. Under the hypothesis of antagonism-mediated balancing selection, SNPS with low P-values should tend to have higher MAFs in the population under consideration than SNPs with high P-values.

\section{Window-based analyses of balancing selection}


We performed genome-wide sliding window analyses (1,000bp windows, $500 \mathrm{bp}$ step size) to investigate regional signatures of balancing selection. Tajima's $D$, which compares SNP polymorphism (nucleotide diversity, $\pi$ ) to SNP abundance (Watterson's estimator, $\theta \mathrm{w}$ ), was compared for windows defined as antagonistic (Qvalue $<0.1)$ or non-antagonistic $(Q$-value $\geq 0.1)$ from the set-based analysis (see section 'Defining candidate antagonistic SNPs and regions'). Under the hypothesis that antagonism generates balancing selection, Tajima's D is expected to be elevated in antagonistic windows. We calculated Tajima's D for each comparison population using PopGenome in $\mathrm{R}^{72}$. As in SNP-based analyses, we incorporated estimates of linked selection ${ }^{71}$ (estimated in $1,000 \mathrm{bp}$ windows) by calculating the residuals of a regression of Tajima's D on estimates of linked selection. Since estimates of linked selection were not available for windows on the $\mathrm{X}$ chromosome, we instead used estimates of recombination rate on this chromosome ${ }^{73}$. We then used a generalised linear model (GLM), assuming Gaussian error structure, to compare residual Tajima's D between antagonistic and non-antagonistic windows.

We also tested for another signature of balancing selection, reduced population differentiation. Measures such as F $_{S T}$ are often considered problematic because they do not correct for the dependency of FST on local levels of polymorphism ${ }^{74}$. However, the availability of genome-wide estimates of linked selection in $D$. melanogaster allowed us to incorporate this confounding variable explicitly. We therefore estimated Fst over windows, using PopGenome, correcting $F_{S T}$ for linked selection in a way analogous to that used for Tajima's D. Since the distribution of FST values is not normally distributed, we contrasted residual FST between antagonistic and non-antagonistic windows using Wilcoxon Rank-Sum tests.

\section{Linkage-based analyses of balancing selection}

670 We examined the extent to which antagonistic haplotypes are selectively maintained by investigating whether antagonistic SNPs have unusually high linkage disequilibrium (LD) in the $\mathrm{ZI}$ population, the population that is most distant from $\mathrm{LH}_{\mathrm{M}}$ and where levels of LD between antagonistic SNPs should be weakest in the absence of long-term balancing selection. Thus, for all SNPs situated within 1000bp 
inferred to be either antagonistic or non-antagonistic), we calculated pairwise LD $\left(\mathrm{r}^{2}\right)$ in PLINK. We then compared $r^{2}$ values between pairs of antagonistic SNP and two control pairs: non-antagonistic pairs, and 'mixed' pairs (antagonistic/nonantagonistic). Comparing pairs of antagonistic SNPs to the mixed pairs allowed us to consider only SNPs located close to an antagonistic SNP, thus effectively controlling for possible non-random distributions of antagonistic pairs and non-antagonistic pairs with respect to genome-wide recombination rates.

To test for significant differences in LD between antagonistic pairs and the two control pairs, we modelled variation in $\mathrm{r}^{2}$ as a declining exponential function of chromosomal distance, and assessed differences in residual $\mathrm{r}^{2}$ (once distance was regressed out) using Wilcoxon Rank-Sum tests.

\section{Statistical software}

690 All statistical analyses were carried out in RStudio (version 1.0.136 ${ }^{75}$ ).

\section{Data availability}

Phenotypic data will be deposited on Dryad prior to publication.

Population genomic data from $\mathrm{LH}_{\mathrm{M}}$ is available at https://zenodo.org/record/159472.

695 Population genomic data from the DGRP, ZI and SA is available at http://www.johnpool.net/genomes.html.

\section{Code availability}

Analysis code is available on request. 


\section{References}

1. Bonduriansky, R. \& Chenoweth, S. F. Intralocus sexual conflict. Trends Ecol. Evol. 24, 280-288 (2009).

705 2. Van Doorn, G. S. Intralocus sexual conflict. Ann. N. Y. Acad. Sci. 1168, 52-71 (2009).

3. Rice, W. R. Sex chromosomes and the evolution of sexual dimorphism. Evolution 38, 735-742 (1984).

4. Cox, R. M. \& Calsbeek, R. Sexually antagonistic selection, sexual dimorphism, and the resolution of intralocus sexual conflict. Am. Nat. 173, 176-187 (2009).

5. Pennell, T. M. \& Morrow, E. H. Two sexes, one genome: The evolutionary dynamics of intralocus sexual conflict. Ecol. Evol. 3, 1819-1834 (2013).

6. Mokkonen, M. et al. Negative frequency-dependent selection of sexually antagonistic alleles in Myodes glareolus. Science 334, 972-974 (2011).

715 7. Stulp, G., Kuijper, B., Buunk, A. P., Pollet, T. V \& Verhulst, S. Intralocus sexual conflict over human height. Biol. Lett. 8, 976-8 (2012).

8. Tarka, M., Åkesson, M., Hasselquist, D. \& Hansson, B. Intralocus sexual conflict over wing length in a wild migratory bird. Am. Nat. 183, 62-73 (2014).

9. Svensson, E. I., McAdam, A. G. \& Sinervo, B. Intralocus sexual conflict over immune defense, gender load, and sex-specific signaling in a natural lizard population. Evolution 63, 3124-3135 (2009).

10. Rice, W. R. Sexually antagonistic genes: experimental evidence. Science 256, 1436-1439 (1992).

11. Berger, D., Berg, E. C., Widegren, W., Arnqvist, G. \& Maklakov, A. A. Multivariate intralocus sexual conflict in seed beetles. Evolution 68, 3457-3469 (2014).

12. Roberts, R. B., Ser, J. R. \& Kocher, T. D. Sexual conflict resolved by invasion of a novel sex determiner in Lake Malawi cichlid fishes. Science 326, 9981001 (2009).

730 13. Barson, N. J. et al. Sex-dependent dominance at a single locus maintains variation in age at maturity in salmon. Nature 528, 405-408 (2015).

14. Delph, L. F. et al. Environment-dependent intralocus sexual conflict in a dioecious plant. New Phytol. 192, 542-552 (2011).

15. Kidwell, J. F., Clegg, M. T., Stewart, F. M. \& Prout, T. Regions of stable 
equilibria for models of differential selection in the two sexes under random mating. Genetics 85, 171-183 (1977).

16. Innocenti, P. \& Morrow, E. H. The sexually antagonistic genes of Drosophila melanogaster. PLoS Biol. 8, e1000335 (2010).

17. Lucotte, E. A., Laurent, R., Heyer, E., Ségurel, L. \& Toupance, B. Detection of allelic frequency differences between the sexes in humans: a signature of sexually antagonistic selection. Genome Biol. Evol. 8, 1489-1500 (2016).

18. Chippindale, A. K., Gibson, J. R. \& Rice, W. R. Negative genetic correlation for adult fitness between sexes reveals ontogenetic conflict in Drosophila. Proc. Natl. Acad. Sci. U. S. A. 98, 1671-1675 (2001).

19. Rice, W. R. et al. Inter-locus antagonistic coevolution as an engine of speciation: assessment with hemiclonal analysis. Proc. Natl. Acad. Sci. U. S. A. 102, 6527-6534 (2005).

20. Gilks, W. P., Pennell, T. M., Flis, I., Webster, M. T. \& Morrow, E. H. Whole genome resequencing of a laboratory-adapted Drosophila melanogaster population sample. F1000Research 5, 2644 (2016).

21. Locke, A. E. et al. Genetic studies of body mass index yield new insights for obesity biology. Nature 518, 197-206 (2015).

22. Price, A. L., Zaitlen, N. A., Reich, D. \& Patterson, N. New approaches to population stratification in genome-wide association studies. Nature Reviews Genetics 11, 459-463 (2010).

23. Bergland, A. O., Behrman, E. L., O’Brien, K. R., Schmidt, P. S. \& Petrov, D. A. Genomic evidence of rapid and stable adaptive oscillations over seasonal time scales in Drosophila. PLoS Genet. 10, e1004775 (2014).

24. Speed, D., Cai, N., Johnson, M. R., Nejentsev, S. \& Balding, D. J.

Reevaluation of SNP heritability in complex human traits. Nat. Genet. 49, 986992 (2017).

25. Yang, J. et al. Genome partitioning of genetic variation for complex traits using common SNPs. Nat. Genet. 43, 519-525 (2011).

26. Purcell, S. et al. PLINK: A tool set for whole-genome association and population-based linkage analyses. Am. J. Hum. Genet. 81, 559-575 (2007).

27. Langley, C. H. et al. Genomic variation in natural populations of Drosophila melanogaster. Genetics 192, 533-598 (2012).

28. Mank, J. E., Hultin-Rosenberg, L., Zwahlen, M. \& Ellegren, H. Pleiotropic 
constraint hampers the resolution of sexual antagonism in vertebrate gene expression. Am. Nat. 171, 35-43 (2008).

29. Yanai, I. et al. Genome-wide midrange transcription profiles reveal expression level relationships in human tissue specification. Bioinformatics 21, 650-659 (2005).

30. He, X. \& Zhang, J. Toward a molecular understanding of pleiotropy. Genetics 173, 1885-1891 (2006).

31. Gavrilets, S. \& Rice, W. R. Genetic models of homosexuality: generating testable predictions. Proceedings of the Royal Society B: Biological Sciences 273, 3031-3038 (2006).

32. Mullon, C., Pomiankowski, A. \& Reuter, M. The effects of selection and genetic drift on the genomic distribution of sexually antagonistic alleles. Evolution 66, 3743-3753 (2012).

33. Connallon, T. \& Clark, A. G. Balancing selection in species with separate sexes: Insights from Fisher's geometric model. Genetics 197, 991-1006 (2014).

34. Mackay, T. F. C. et al. The Drosophila melanogaster Genetic Reference Panel. Nature 482, 173-178 (2012).

35. Huang, W. et al. Natural variation in genome architecture among 205 Drosophila melanogaster Genetic Reference Panel lines. Genome Res. 24, 1193-1208 (2014).

36. Duchen, P., Živković, D., Hutter, S., Stephan, W. \& Laurent, S. Demographic inference reveals African and European admixture in the North American Drosophila melanogaster population. Genetics 193, 291-301 (2013).

37. Lack, J. B. et al. The Drosophila Genome Nexus: A population genomic resource of 623 Drosophila melanogaster genomes, including 197 from a single ancestral range population. Genetics 199, 1229-1241 (2015).

38. Lack, J. B., Lange, J. D., Tang, A. D., Corbett-Detig, R. B. \& Pool, J. E. A thousand fly genomes: An expanded Drosophila Genome Nexus. Mol. Biol. Evol. 33, 3308-3313 (2016).

39. Kelly, J. K. A test of neutrality based on interlocus associations. Genetics 146, 1197-1206 (1997).

40. Lachaise, D. et al. Historical biogeography of the Drosophila melanogaster species subgroup. Evol. Biol. 22, 159-225 (1988). 
41. Li, H. \& Stephan, W. Inferring the demographic history and rate of adaptive substitution in Drosophila. PLoS Genet. 2, e1004775 (2006).

42. Thornton, K. \& Andolfatto, P. Approximate Bayesian inference reveals evidence for a recent, severe bottleneck in a Netherlands population of Drosophila melanogaster. Genetics 172, 1607-1619 (2006).

43. Connallon, T. \& Hall, M. D. Genetic correlations and sex-specific adaptation in changing environments. Evolution 70, 2186-2198 (2016).

44. Pool, J. E. et al. Population Genomics of Sub-Saharan Drosophila melanogaster: African Diversity and Non-African Admixture. PLoS Genet. 8, e1003080 (2012).

45. Charlesworth, D. Balancing selection and its effects on sequences in nearby genome regions. PLoS Genet. 2, e64 (2006).

815 46. Asthana, S., Schmidt, S. \& Sunyaev, S. A limited role for balancing selection. Trends in Genetics 21, 30-32 (2005).

47. Poissant, J., Wilson, A. J. \& Coltman, D. W. Sex-specific genetic variance and the evolution of sexual dimorphism: A systematic review of cross-sex genetic correlations. Evolution 64, 97-107 (2010).

48. Connallon, T. \& Clark, A. G. Evolutionary inevitability of sexual antagonism. Proc. R. Soc. B Biol. Sci. 281, 2013-2123 (2014).

49. Connallon, T. \& Clark, A. G. Sex linkage, sex-specific selection, and the role of recombination in the evolution of sexually dimorphic gene expression. Evolution 64, 3417-3442 (2010).

825 50. Stewart, A. D., Pischedda, A. \& Rice, W. R. Resolving intralocus sexual conflict: genetic mechanisms and time frame. J. Hered. 101, S94-9 (2010).

51. Fry, J. D. The genomic location of sexually antagonistic variation: Some cautionary comments. Evolution 64, 1510-1516 (2010).

52. Arnqvist, G., Vellnow, N. \& Rowe, L. The effect of epistasis on sexually antagonistic genetic variation. Proc. R. Soc. B Biol. Sci. 281, 20140489 (2014).

53. Rice, W. R. et al. Inter-locus antagonistic coevolution as an engine of speciation: assessment with hemiclonal analysis. Proc. Natl. Acad. Sci. U. S. A. 102, 6527-6534 (2005).

54. Abbott, J. K. \& Morrow, E. H. Obtaining snapshots of genetic variation using hemiclonal analysis. Trends Ecol. Evol. 26, 359-368 (2011).

55. Danecek, P. et al. The variant call format and VCFtools. Bioinformatics 27, 
2156-2158 (2011).

56. Speed, D., Hemani, G., Johnson, M. R. \& Balding, D. J. Improved heritability estimation from genome-wide SNPs. Am. J. Hum. Genet. 91, 1011-1021

840 (2012).

57. Hadfield, J. D. MCMC methods for multi-response generalized linear mixed models: the MCMCglmm R package. J. Stat. Softw. 33, 1-22 (2010).

58. Yang, J. et al. Common SNPs explain a large proportion of heritability for human height. Nat. Genet. 42, 565-569 (2010).

59. Astle, W. \& Balding, D. J. Population structure and cryptic relatedness in genetic association studies. Stat. Sci. 24, 451-471 (2009).

60. Yang, J. et al. Genomic inflation factors under polygenic inheritance. Eur. J. Hum. Genet. 19, 807-812 (2011).

61. Aulchenko, Y. S., Stephan, R., Isaacs, A. \& van Duijn, C. M. GenABEL: an R library for genome-wide association analysis. Bioinformatics 23, 1294-1296 (2007).

62. Casper, J. et al. The UCSC Genome Browser database: 2018 update. Nucleic Acids Res. 46, D762-D769 (2018).

63. McLaren, W. et al. Deriving the consequences of genomic variants with the Ensembl API and SNP Effect Predictor. Bioinformatics 26, 2069-2070 (2010).

64. Cabrera, C. P. et al. Uncovering networks from genome-wide association studies via circular genomic permutation. G3 Genes, Genomes, Genet. 2, 1067-1075 (2012).

65. Eden, E., Navon, R., Steinfeld, I., Lipson, D. \& Yakhini, Z. GOrilla: a tool for discovery and visualization of enriched $\mathrm{GO}$ terms in ranked gene lists. BMC Bioinformatics 10, 48 (2009).

66. Gnad, F. \& Parsch, J. Sebida: a database for the functional and evolutionary analysis of genes with sex-biased expression. Bioinformatics 22, 2577-2579 (2006).

865 67. Chintapalli, V. R., Wang, J. \& Dow, J. A. T. Using FlyAtlas to identify better Drosophila melanogaster models of human disease. Nat. Genet. 39, 715-720 (2007).

68. Gramates, L. S. et al. FlyBase at 25: Looking to the future. Nucleic Acids Res. 45, D663-D671 (2017).

870 69. Keane, J. A. et al. SNP-sites: rapid efficient extraction of SNPs from multi- 
FASTA alignments. Microb. Genomics 2, e000056 (2016).

70. Turchin, M. C. et al. Evidence of widespread selection on standing variation in Europe at height-associated SNPs. Nat. Genet. 44, 1015-1019 (2012).

71. Elyashiv, E. et al. A genomic map of the effects of linked selection in Drosophila. PLoS Genet. 12, e1006130 (2016).

72. Pfeifer, B., Wittelsburger, U., Ramos-Onsins, S. E. \& Lercher, M. J. PopGenome: An efficient swiss army knife for population genomic analyses in R. Mol. Biol. Evol. 31, 1929-1936 (2014).

73. Comeron, J. M., Ratnappan, R. \& Bailin, S. The many landscapes of recombination in Drosophila melanogaster. PLoS Genet. 8, e1002905 (2012).

74. Cruickshank, T. E. \& Hahn, M. W. Reanalysis suggests that genomic islands of speciation are due to reduced diversity, not reduced gene flow. Mol. Ecol. 23, 3133-3157 (2014).

75. RStudio Team. RStudio: Integrated Development for R. RStudio. RStudio, Inc., Boston, MA (2015). Available at: https://www.rstudio.com/.

\section{Acknowledgements}

We are grateful to Aida Andrés for her suggestions on the analysis of balancing selection and to Laurent Keller, Andrew Pomiankowski and François Balloux for 890 helpful comments on earlier versions of the manuscript. FR was funded by a London NERC DTP studentship (NERC grant NE/L002485/1), MSH was funded by a UCL IMPACT PhD studentship, EHM, TMP, IF and FCI by a European Research Council Grant (280632) and a Royal Society University Research Fellowship and MR and KF by a Natural Environment Research Council research grant (NE/G0189452/1).

\section{Author contributions}

EHM, MR, KF, MSH and FR conceived the project; TMP, IF and EHM conducted phenotypic experiments; FR, MSH, MR and FCl analysed the data; FR, MR, MSH and KF wrote the manuscript.

900

\section{Competing financial interests}

The authors declare no competing interests. 

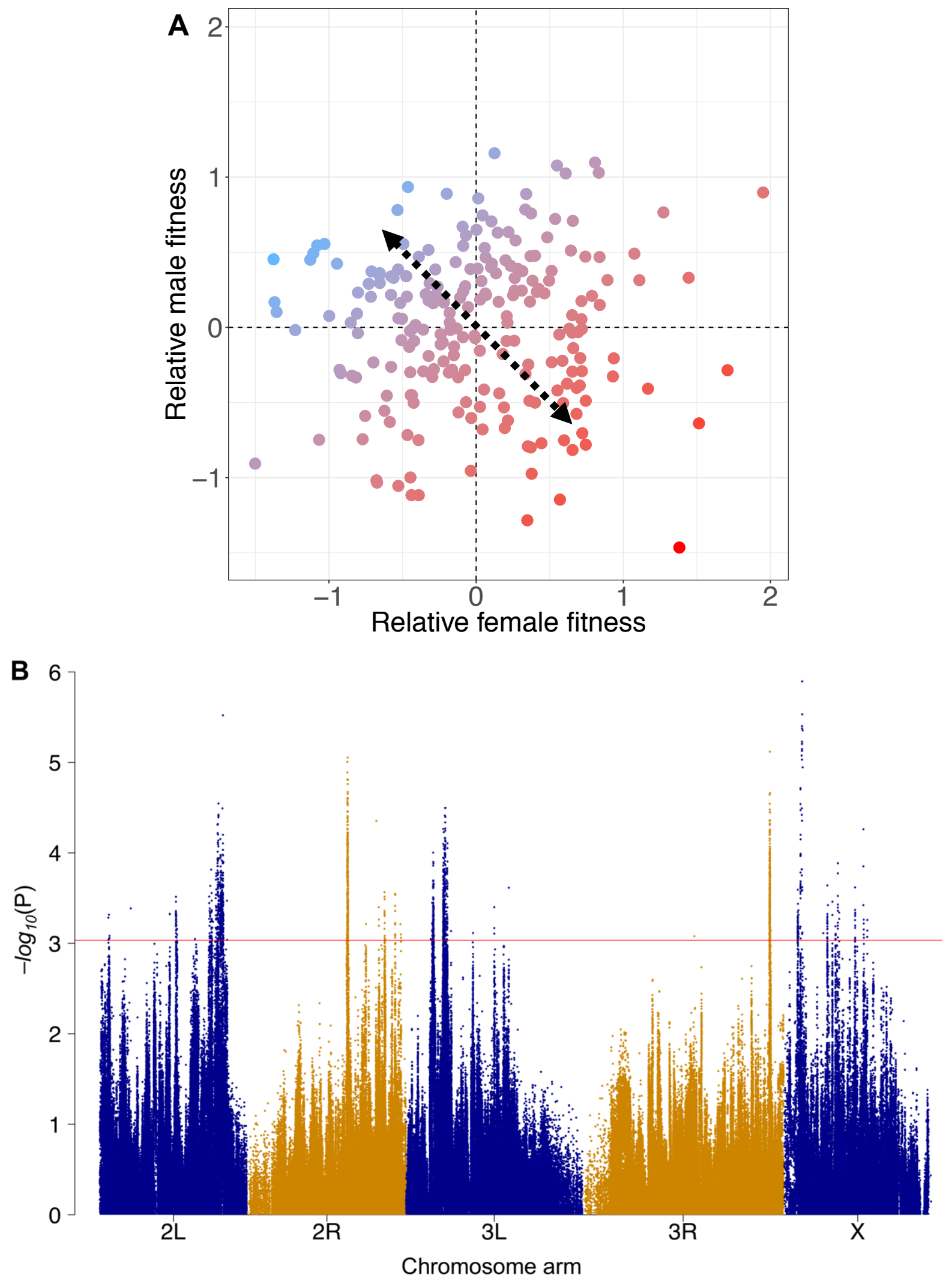
Figure 1. Genome-wide association mapping of sexual antagonism. A. Relative

910 male and female lifetime reproductive fitness estimates for 223 D. melanogaster hemiclonal lines. Fitness measures have been scaled to be normally distributed. Colours denote each line's antagonism index, i.e. their position along a spectrum (arrows) ranging from male-beneficial, female-detrimental fitness effects (blue), to female-beneficial, male-detrimental effects (red). B. Association of each SNP with

915 the antagonism index along the five major $D$. melanogaster chromosome arms, presented as a Manhattan plot where each point represents the $-\log _{10}(P)$ value from a Wald $\chi^{2}$ association test. Colours denote chromosome arms, the horizontal line represents the $Q$-value cut-off $(0.3)$ used to define candidate antagonistic SNPs. 
A
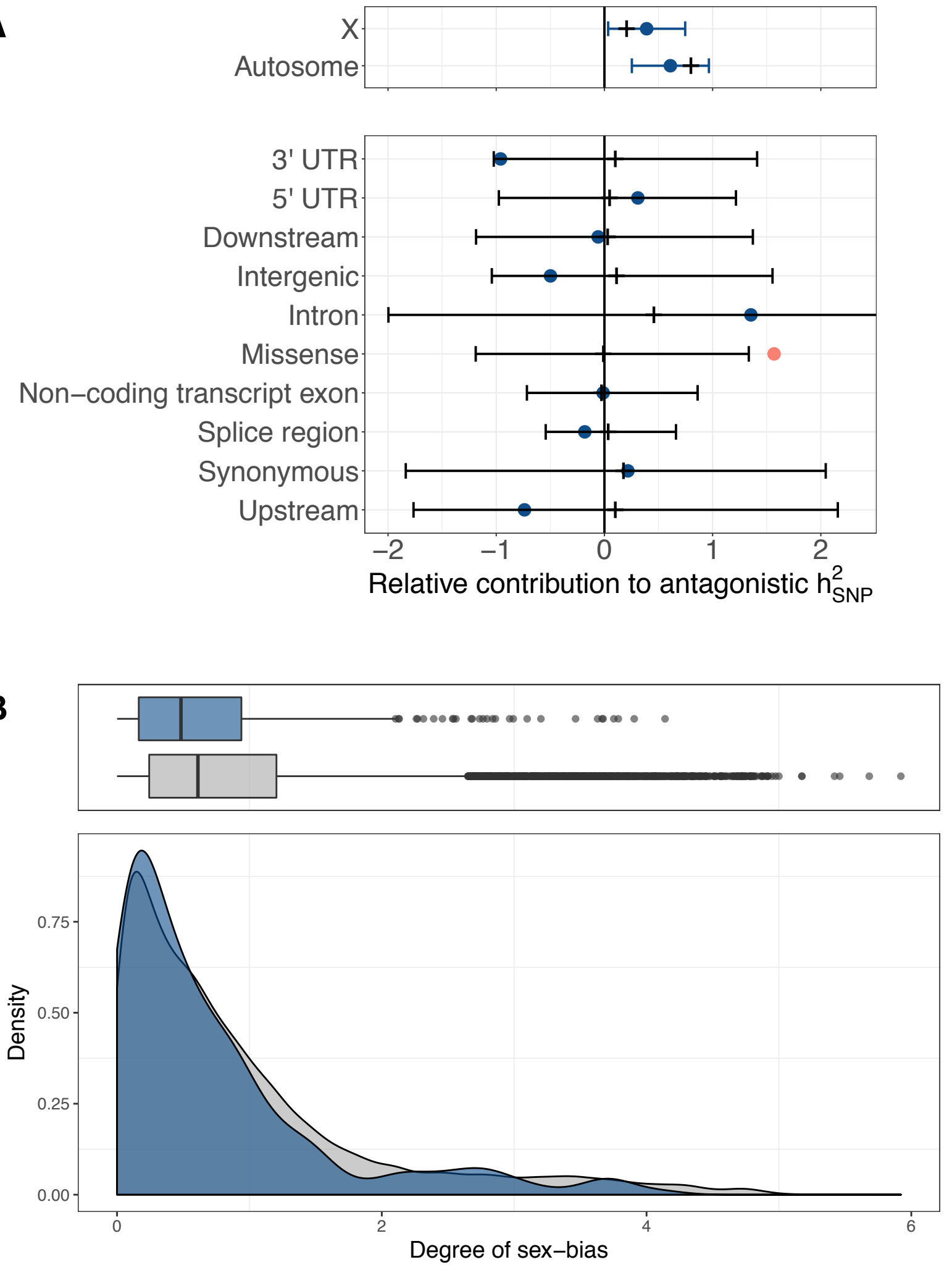


\section{Figure 2. Genomic distribution and functional characteristics of antagonistic}

variants. A. Relative contribution (proportional share) of different chromosomal compartments (top) and functional categories (bottom) to total antagonistic SNP heritability $\left(h_{S N P}^{2}\right)$. Dots represent estimated $h_{S N P}^{2}$ contributions $( \pm 95 \% \mathrm{Cl}$, for chromosomal compartments), with colours indicating significant under or overrepresentation (red: P-value<0.05; blue: P-value>0.05). Expected $h_{S N P}^{2}$ contributions are presented either as black notches (fixed values for chromosomal compartments) or mean $\pm 95 \% \mathrm{Cl}$ of the empirical null distribution computed through permutation (for functional categories). See Methods for additional details. B. Distributions of the absolute degree of sex-biased expression for antagonistic (blue) and nonantagonistic (grey) genes. 

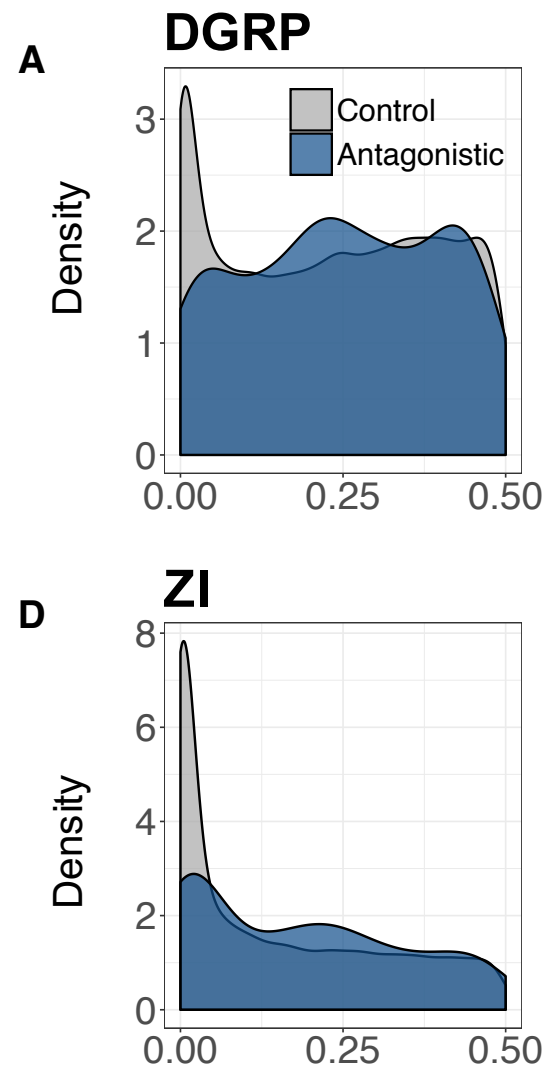

Minor allele frequency

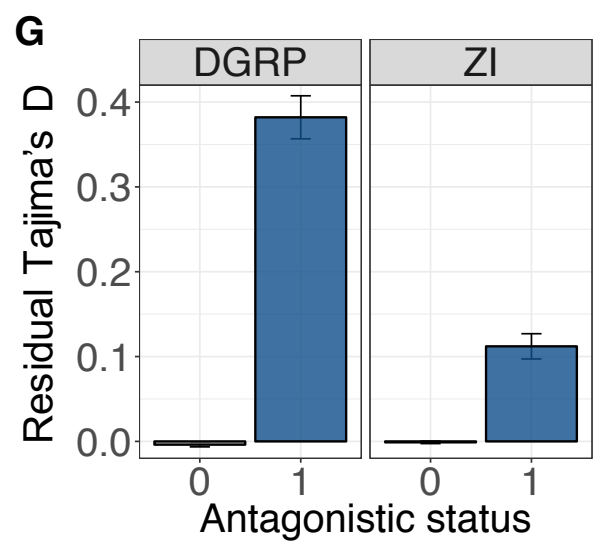

B

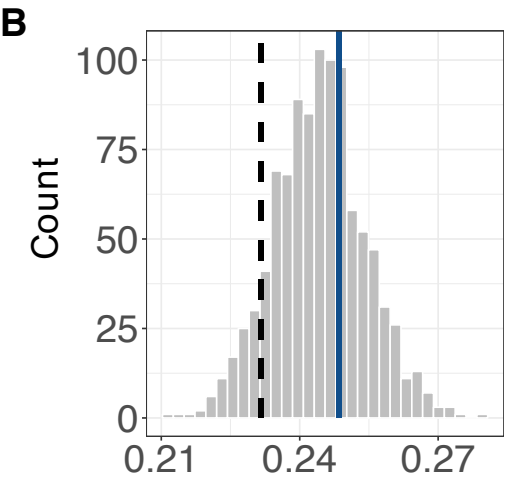

E

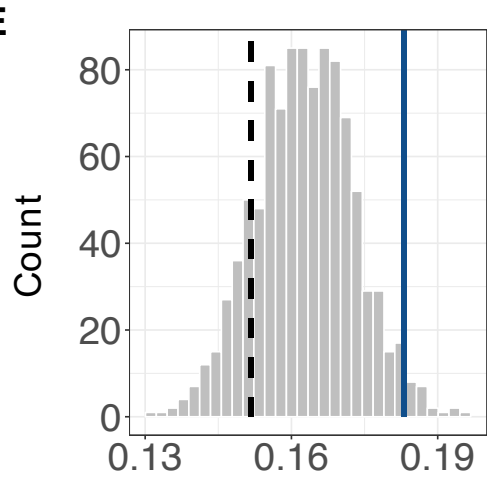

Minor allele frequency

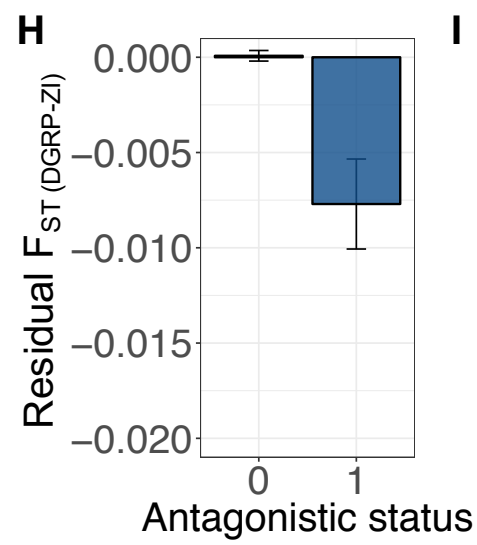

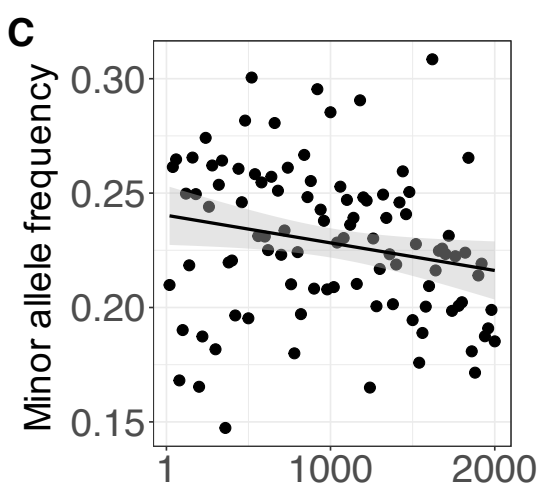

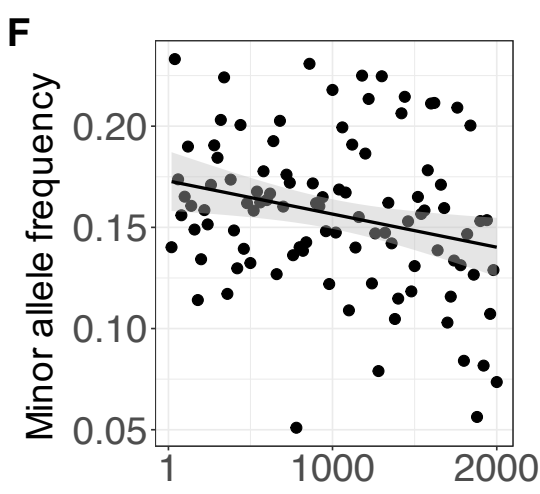

P-value order

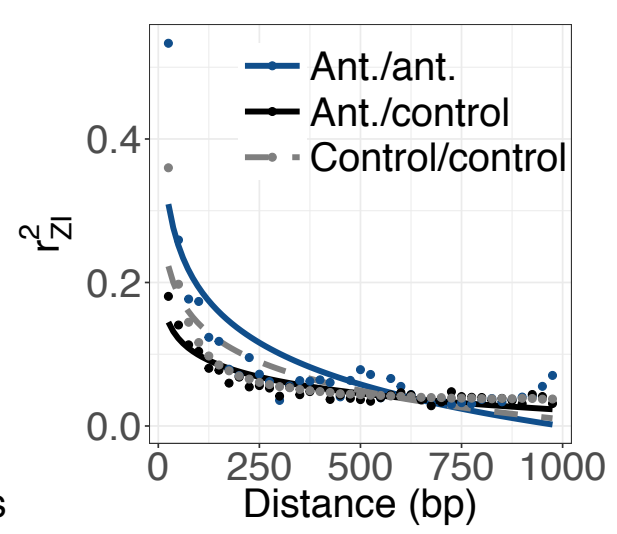




\section{Figure 3. Signatures of balancing selection associated with antagonistic}

population). A,D. Spectra of raw minor allele frequencies (MAF) for LD-pruned antagonistic (blue) and non-antagonistic ('control', grey) SNPs in the DGRP and ZI populations. B,E. Distribution of mean MAFs for 1,000 sets of LD-independent, nonantagonistic SNPs that have been frequency-matched to $\mathrm{LH}_{\mathrm{M}}$ antagonistic SNPs

955 (see Methods). Blue line denotes mean MAF of antagonistic SNPs; black dashed line denotes mean MAF of non-antagonistic SNPs before frequency-matching. $\mathbf{C , F}$. MAF in the DGRP and ZI populations across 100 sets LD-independent SNPs, each set matched for $\mathrm{LH}_{\mathrm{M}}$ allele frequencies, and presented in ascending order by $\mathrm{P}$ value. For visualisation purposes, a linear regression line $( \pm 95 \% \mathrm{Cl})$ is shown. $\mathbf{G}$.

960 Mean ( \pm S.E.) residual Tajima's D (corrected for linked selection, see Methods) for antagonistic windows (blue; 'antagonistic status=1') and non-antagonistic windows (grey; 'antagonistic status=0') in the DGRP and ZI populations. H. Residual F ( \pm S.E.), corrected for linked selection (see Methods), for antagonistic and nonantagonistic windows in the DGRP and ZI populations. I. Linkage disequilibrium $\left(r^{2}\right)$ in the ZI population between pairs of antagonistic SNPs (blue, 'Ant./ant.'), pairs of non-antagonistic SNPs (grey, 'Control/control') and mixed pairs (black, 'Ant./control'). Points represent mean $r^{2}$ across $25 b p$ bins; $r^{2}$ is modelled as a declining exponential function of distance (fitted lines). 